\title{
Diseño y transferencia del aprendizaje en un curso masivo abierto a distancia
}

Nohemi Rivera Vázquez', María Soledad Ramírez Montoya², Diana Hernández Montoya ${ }^{3}$ \& Ana María Sandoval Poveda ${ }^{4}$

1. Asistente de investigación de la Escuela Nacional de Educación, Humanidades y Ciencias Sociales del Tecnológico de Monterrey, México; nohemirv@hotmail.com

2. Profesora-investigadora de la Escuela Nacional de Educación, Humanidades y Ciencias Sociales del Tecnológico de Monterrey, México. Investigadora Nacional del Sistema Nacional de Investigadores y Titular de la Cátedra UNESCO/ICDE para el movimiento educativo abierto en América Latina; solramirez@itesm.mx

3. Investigadora y gestora de proyectos en áreas de educación, tecnología e innovación, Vicerrectoría de Investigación, UNED, Costa Rica; dhernandez@uned.ac.cr

4. Productora académica e investigadora, UNED, Costa Rica; amsandoval@uned.ac.cr

\section{Resumen}

Este estudio forma parte del Programa de Intercambio Académico de la Asociación Nacional de Universidades e Instituciones de Educación Superior (ANUIES) y el Consejo Superior Universitario Centroamericano (CSUCA) en el marco del proyecto bilateral Cooperación interuniversitaria para la formación e investigación en el movimiento educativo abierto. Su objetivo fue analizar la percepción de los participantes del curso masivo abierto a distancia (MOOC, por sus siglas en inglés) "Innovación educativa con recursos abiertos" en cuanto a su diseño y las posibilidades de transferencia, para lo cual se utilizó un método mixto con la aplicación de seis instrumentos. Se encontró que los aprendices perciben que pudieron transferir los aprendizajes a su práctica docente y valoran la socialización, la comunicación, la retroalimentación y el apoyo de los facilitadores.

Palabras clave: aprendizaje, educación masiva, curso de enseñanza, recursos educativos abiertos, educación a distancia.

\section{Abstract \\ Instructional design and learning transfer in a Massive Open Online Courses (MOOC)}

This study is part of the Academic Exchange Program of the National Association of Universities and Institutions of Higher Education (ANUIES, for its acronym in Spanish) and the Higher University Council of Central America (CSUCA, for its acronym in Spanish) under the bilateral university cooperation project for training and research in the open education movement. Its aim was to analyze the perception of the "Educational innovation with open resources" Massive Open Online Courses (MOOC) participants in terms of course design and transfer possibilities. We used a mixed method approach, applying six different instruments. We found that socialization, communication, feedback and support from facilitators are highly valued by learners of a MOOC. In addition, participants perceived they could transfer the lessons learned during the course to their teaching practice.

Key words: learning, mass education, educational courses, open educational resources, distance education. 


\section{INTRODUCCIÓN}

Los ambientes de aprendizaje se potencian con las nuevas tecnologías y amplían su naturaleza de presencialidad a la distancia. Esto implica cambios que requieren la evaluación de los modelos pedagógicos. Además, los individuos y las organizaciones del sector educativo se deben mantener informadas y plantearse nuevos enfoques educativos que satisfagan las necesidades emergentes (Siemens, 2008). Desde esta perspectiva, uno de los desafíos es buscar opciones que apoyen a los individuos en su aprendizaje (favoreciendo su interacción, conexiones y colaboraciones), integren el uso de recursos variados y respondan a estos nuevos escenarios.

La variación en los ambientes de aprendizaje apoya la formación de comunidades académicas con opciones diferenciadas; cada vez más docentes usan redes sociales, seminarios en línea (webinars), y comunidades de aprendizaje como herramientas para su desarrollo profesional (Cavanagh, 2013). En este contexto de información y formación autodidacta, los MOOC (Massive Open Online Courses) facilitan el desarrollo del conocimiento compartido a través de un diálogo continuo que invita a la participación abierta alrededor de un tema (Kop, Fournier y Mak, 2011). Esta modalidad potencia el intercambio de recursos y la construcción de conexiones para la formación de comunidades de aprendizaje.

En algunos diseños de $\mathrm{MOOC}$ se puede encontrar un enfoque centrado en fomentar pensamiento crítico, colaboración y autorregulación entre los participantes; así como el desarrollo de competencias y el uso de las tecnologías de la información y comunicación (TIC) para crear y publicar recursos. Los diseños de cursos futuros deben considerar qué aspectos de los MOOC pueden igualar o superar las condiciones pedagógicas de los cursos virtuales tradicionales (Roig, Mengual y Suárez, 2014).

Por lo anterior, es necesario plantear rutas de investigación que abran espacios de reflexión sobre el impacto del diseño de los MOOC en el aprendizaje de los participantes, la formación de redes y la transferencia del conocimiento a su ambiente laboral; entendida como el hecho de que la persona que aprende ponga en práctica lo asimilado en nuevas situaciones (Wenzelburger, 1987). En este sentido, la presente investigación se enfoca en responder a las siguientes interrogantes: ¿cuáles son las percepciones de los participantes con respecto al diseño del curso?, ¿consideran los participantes que podrían transferir a su práctica diaria los aprendizajes obtenidos en el curso?

El término participantes hace referencia a quienes se inscribieron en el curso. El presente estudio surge en el marco de la convocatoria 2013 del Programa de Intercambio Académico ANUIES-CSUCA, financiado por la Secretaría General Iberoamericana (SEGIB) a través de la Secretaría de Relaciones Exteriores (SER) de México. Allí se evaluaron los proyectos (especialistas de México y Centroamérica) y se aprobó el proyecto bilateral E-9 Cooperación interuniversitaria para la formación e investigación en el movimiento educativo abierto. Este proyecto se llevó a cabo por profesores investigadores del Tecnológico de Monterrey (México) y la Universidad Estatal a Distancia (Costa Rica), quienes diseñaron este estudio, construyeron los instrumentos, facilitaron los procesos en el MOOC y condujeron la investigación.

El MOOC del estudio, denominado Innovación educativa con recursos abiertos, pretende que los participantes adquirieran competencias para el uso de Recursos Educativos Abiertos (REA), que les permitan contribuir, con prácticas innovadoras, al movimiento educativo abierto. El diseño del curso abarcó cuatro semanas y se plantearon estrategias de conectivismo, portafolios digitales, socialización del conocimiento con grupos de autoestudio, expertos invitados y recursos educativos abiertos. Además cuenta con materiales, ebooks de acceso abierto, actividades de autogestión y autoestudio, espacios de socialización, redes sociales, actividades de portafolios digitales, evaluaciones de pares y autoevaluaciones.

El curso fue diseñado e implementado con dos profesores titulares y apoyado (procesos de facilitación) con dos docentes tutores y 2500 voluntarios (TA, siglas en inglés de Teaching Assistant) seleccionados con base en una encuesta diagnóstica. De importancia sustancial fue la participación de los profesores 
investigadores del proyecto ANUIES-CSUCA con miras a dirigir una estrategia metodológica para recopilar los datos del estudio.

\section{REFERENTE TEÓRICO}

\section{Diseño de ambientes de aprendizaje}

Los ambientes de aprendizaje son "espacios" donde se construyen experiencias de aprendizaje. Ramírez (2012) menciona que los ambientes de aprendizaje pueden presentarse en las modalidades presenciales, a distancia (e-learning), multimodales o combinadas, usando la presencialidad y ambientes a distancia (b-learning), o promoviendo el aprendizaje móvil (m-learning). En cada uno de ellos, el diseño de los ambientes debe contemplar finalidades, contenidos, recursos, espacios, comunicación, infraestructura, evaluación, estrategias y sentido de transcendencia. De tal forma que al diseñar un ambiente los diversos componentes deben confluir en una determinada visión de aprendizaje.

\section{Ambientes de aprendizaje MOOC}

En los ambientes de aprendizaje a distancia se han incrementado las posibilidades de diseños, uno de ellos se muestra en los MOOC. El diseño e implementación de un MOOC se basa en cuatro actividades (Kop et al., 2011):

1. El acceso a una gran variedad de recursos,

2. el seguimiento del contenido estudiado (creando un blog, participando en una discusión Moodle),

3. la creación de un recurso de su autoría,

4. el compartir el trabajo.

Lo diverso del diseño de los $\mathrm{MOOC}$ es una de sus características y entre sus ventajas se halla la de presentar una gran variedad de perspectivas alrededor de un tema en particular (Cormier y Siemens, 2010). Los MOOC se entienden como un espacio abierto ya que se desarrollan en varios lugares y poseen pocas limitantes de inscripción y asistencia, lo cual facilita el conocimiento a un grupo mucho más amplio de usuarios, quienes pueden continuar usando el sitio central y conservar los lazos sociales (McAulay, Stewart y Siemens, 2010; Cavanagh, 2013). De hecho, los aprendices pueden retirarse en cualquier momento y organizar su participación de acuerdo con sus propias metas de aprendizaje y habilidades previas. Usualmente, solo unos cuantos individuos participan de manera activa y la mayoría toman un rol pasivo (Esposito, 2012).

\section{Transferencia de aprendizajes en ambientes a distancia}

La consideración de la transferencia como una variable de estudio se trabaja desde los años ochenta y esto se evidencia en la forma que se incluye y estudia en diferentes investigaciones. En esa época se trabajó el concepto como recibir y estudiar un contenido y llevarlo a la aplicación o práctica; se dice que "la transferencia ocurre cuando lo que se aprende en una situación facilita (o inhibe) el aprendizaje o desempeño en otras" (Wenzelburger, 1987, p. 1). 
En el ámbito educativo, el concepto de transferencia de conocimiento se relaciona con la capacidad del ser humano para tomar en cuenta lo aprendido y usarlo para una mayor comprensión de las situaciones que se le presentan, de manera que esto se traduzca en respuestas más completas y precisas a problemas planteados (Contreras, Torres y Ramírez, 2010). En este sentido, en el presente estudio la transferencia y uso del conocimiento se plantean como elementos unidos en el ámbito educativo. En cursos a distancia, como los MOOC, el diseño de ambientes debe contemplar la forma de potenciar el proceso mental que le permite a una persona aplicar sus conocimientos en contextos y situaciones nuevas.

Entre los diversos factores que afectan la transferencia, los investigadores deben contemplar la importancia del diseño del curso. En investigaciones previas se han considerado diversos factores relacionados con el diseño en los modelos de transferencia del aprendizaje de los cursos de capacitación. Por ejemplo, Bhatti y Kaur (2010) encontraron que cuando el aprendiz percibe que el contenido del entrenamiento es similar a sus labores reales, tiende a reaccionar de manera positiva. Aunado a esto, Gegenfurtner (2011) encontró que el tipo de conocimiento, la instrucción y las condiciones de evaluación afectan la motivación de los participantes y, por consiguiente, su aprendizaje y transferencia; además, la correlación era generalmente más positiva en un ambiente de capacitación centrado en el alumno que en un ambiente centrado en el conocimiento.

Es necesario investigar acerca de la influencia del diseño de los cursos en la transferencia de aprendizaje y obtener medidas más directas de los resultados de la transferencia. Explorar qué se transfiere, cuándo, cómo y bajo qué condiciones, y no solo si la transferencia ocurre; colectar múltiples medidas combinando cuestionarios, observaciones y entrevistas para triangular los resultados de la investigación e identificar posibles inconsistencias (Volet, 2013).

\section{METODOLOGÍA}

El método del estudio fue mixto con aplicación de seis instrumentos: cuatro autoevaluaciones de aprendizaje (una por semana durante las cuatro semanas del curso) y dos grupos de enfoque con participantes e instructores (uno en México, otro en Costa Rica). Las categorías de análisis fueron la percepción del diseño del curso y las posibilidades de transferencia de aprendizajes, por parte de los participantes. En paralelo, los participantes permitieron contextualizar esta información al aportar elementos colaterales respecto a sus expectativas y otros temas. El análisis fue exploratorio donde primero se codificaron variables cualitativas para interpretarlas y representarlas gráficamente para una mayor comprensión de la información obtenida (Creswell y Plano, 2011).

Se inscribieron 14822 participantes de 62 países. La población en el curso varió semana a semana, en la primera semana 10725 participantes respondieron la encuesta de inicio. Para el estudio no se usó la inscripción como pauta, pues muchos solo se inscribieron para ver los materiales y no formaron parte del desarrollo del curso. Para la última semana la participación fue de 634 individuos, es decir, alrededor de $6 \%$ de la población inicial. Por tanto la población total del estudio considerada fueron los participantes que respondieron todos los instrumentos. Se definió además que se trabajaría con un margen de error de $5 \%$ y un nivel de confianza de $95 \%$, de manera que se pudiese determinar el tamaño adecuado de la muestra para el estudio (Collins, 2003; Gómez, 2011). En este caso se utilizó la fórmula proporcionada por López (s.f.).

$$
n=\frac{N(0,5 \alpha)^{2}}{1+\left(e^{2}(N-1)\right)}
$$

Donde: $N$ es la población total considerada para el estudio, e corresponde al error elegido y a es el nivel de confianza. Así, se trabajó con una muestra de 239 individuos. 
La recolección y el análisis de los datos cuantitativos y cualitativos fue integrada en las distintas etapas del proceso de investigación (Creswell, Plano, Gutmann y Hanson, 2003). En la primera y en la última autoevaluación (semanas 1 y 4) se les preguntó a los participantes si consideraban que podían darle uso a los REA en su práctica profesional y de qué forma. Los datos se analizaron de manera longitudinal para evaluar si el curso había logrado la tarea de facilitar el uso de REA en la práctica docente (si habían logrado una transferencia del aprendizaje).

Asimismo, se recolectaron datos cualitativos de las autoevaluaciones contestadas al término de cada semana. En ellas se les preguntaba cuál había sido su mayor logro dentro del tema semanal (conocimiento, habilidad, actitud, competencia) y sobre aspectos relacionados con el diseño del curso (actividades, tiempo disponible y espacios de socialización del conocimiento). Finalmente, se organizaron dos reuniones con participantes del MOOC: una en Monterrey (México) y otra en San José (Costa Rica). En la primera estuvieron 20 profesores del Tecnológico de Monterrey que participaban en el MOOC, algunos de manera presencial en la sala y otros a través de la herramienta Hangout de Google (4 y 16 respectivamente). En la segunda hubo nueve participantes costarricenses. En los encuentros se examinaron las percepciones de los participantes respecto a los contenidos del curso, el diseño de las actividades y las evaluaciones, así como los espacios para la comunicación e interacción entre el grupo y sus tutores. Al final se brindaron algunas recomendaciones de mejora que pudieran facilitar la transferencia.

Los datos cualitativos recolectados se analizaron por medio de la comparación constante para proponer una teoría que explique cómo perciben los alumnos el diseño pedagógico del MOOC y la transferencia de sus aprendizajes, así como la relación que existe entre el diseño y la transferencia de nuevos conocimientos y habilidades (De la Cuesta, 2006; Sandoval, 2002; Strauss y Corbin, 1997).

La información de los instrumentos se trabajó mediante hojas de cálculo y en el Programa de estadística para ciencias sociales Statistical Package for the Social Sciences (SPSS) versión 20. Los datos se analizaron estadísticamente y por sumas categóricas de incidencia, según la naturaleza de los instrumentos, mediante gráficas, tablas y fórmulas.

Con base en los datos, las secuencias iterativas de análisis cuantitativos y cualitativos complementaron los resultados derivando interpretaciones razonadas. Creswell, Klassen, Plano y Smith (2011) aluden a que existe un punto en las iteraciones donde se suscita una interferencia de los métodos, lo cual puede corroborar o no los hallazgos en cada fase. Fue vital entonces centrar el enfoque con mayor peso y fundamentarse en el objetivo del estudio para realizar interpretaciones.

\section{RESULTADOS}

Con el fin de presentar datos triangulados para la interrogante: ¿cuáles son las percepciones de los participantes con respecto al diseño del curso?, se presentan los resultados de autoevaluaciones obtenidos en diferentes momentos del MOOC.

\section{Socialización}

En dos momentos del curso (autoevaluaciones de las semanas 1 y 4), se consultó por los espacios de socialización del conocimiento que les permitieron aprender. Al respecto, una de las participantes compartió en la sesión presencial de Costa Rica lo siguiente: "Sugiero que el día que se entreguen materiales por lo menos den un día más para compartir con los compañeros los materiales y mejorarlos", ejemplo del uso de los espacios encontrados para esta labor. En ambos momentos, los participantes coincidieron en que los espacios fueron los foros y las redes sociales; $70 \%$ los señaló como los puntos preferidos al 
comenzar el curso. Además, al finalizar el curso, 58\% indicó que usaba los foros y $52 \%$ que hacía uso del Facebook. La cantidad de personas que eligió estas opciones evidencia que, respecto al diseño, los espacios de socialización cumplieron su objetivo.

\section{Evaluación}

En dos momentos del curso (autoevaluaciones de las semanas 2 y 4, AA2 y AA4, respectivamente), se consultó a los participantes si consideraban que la evaluación en el MOOC valoraba los nuevos aprendizajes adquiridos y por qué. En ambos momentos, la mayoría consideraron que sí, la primera vez casi tres cuartas partes lo hicieron y la segunda más de $60 \%$. Entre las principales razones que estimaron para responder afirmativamente se encuentran las siguientes:

"... ayuda mucho la evaluación entre pares".

“... especialmente en los portafolios solicitados se puede evidenciar la integración de los conocimientos adquiridos por los diversos medios que se nos presentaron".

"... pues el sistema es participativo e interactivo entre los que llevamos el curso".

"El último portafolio realmente me ayudó porque es otra forma diferente de como uno está acostumbrado a planear".

En las expresiones anteriores los participantes mencionan dos de las estrategias empleadas para evaluar las actividades del curso, como positivas: la evaluación entre pares y los portafolios.

La información apoya la forma en la que se planteó la evaluación. En las figuras 1 y 2 se presenta la distribución de las respuestas dadas por los participantes en las autoevaluaciones que se aplicaron en las semanas 2 y 4, en las que se les hizo la pregunta: ¿la evaluación valora los aprendizajes adquiridos? Son estos datos los que apoyan las afirmaciones dadas al respecto.

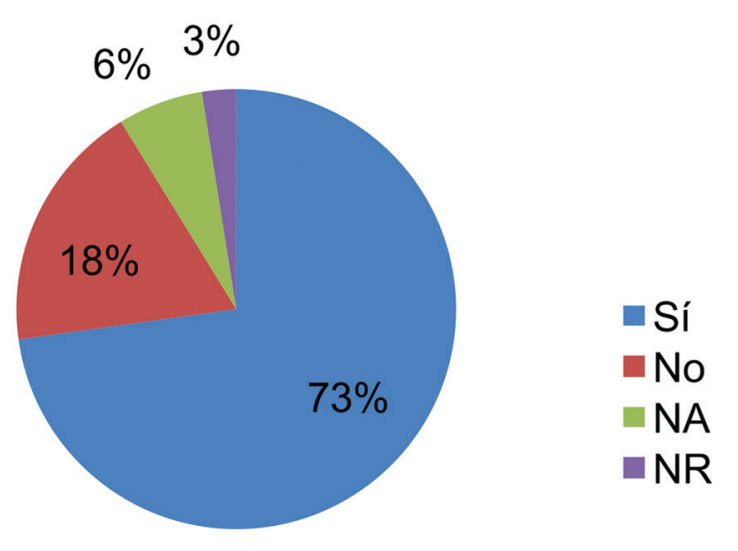

Figura 1. ¿La evaluación valora los aprendizajes adquiridos?

Fuente: elaboración propia basada en los datos proporcionados por los participantes. 


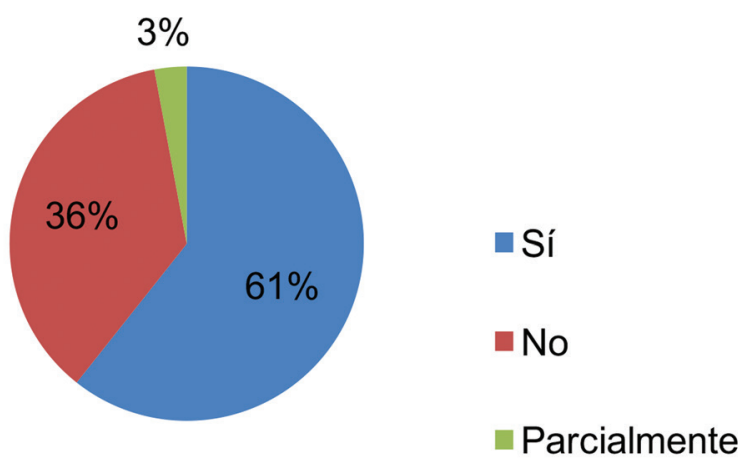

Figura 2. ¿La evaluación valora los aprendizajes adquiridos? (AA4).

Fuente: elaboración propia basada en los datos proporcionados por los participantes.

\section{Intervenciones y actividades de los facilitadores}

Con el fin de conocer las percepciones de los participantes con respecto al diseño del curso, se les consultó, en dos oportunidades, acerca de las intervenciones de los facilitadores para apoyar el aprendizaje. Esto se hizo en dos autoevaluaciones AA2 y AA4, es decir durante la segunda y la cuarta semana; los correspondientes resultados se resumen en las figuras 3 y 4 .

Desde el punto de vista de los participantes, los facilitadores se dedicaron a motivar el proceso y a ofrecer elementos para ordenar la tarea. Sin embargo, otras intervenciones como proponer estrategias y comentar el proceso, también fueron consideradas por los participantes. El dinamismo impuesto por el horario y los facilitadores hizo que las apreciaciones de los participantes dejaran entrever la calidad de su trabajo. Lo anterior se evidencia en ambas autoevaluaciones donde los participantes valoran la tarea de los facilitadores como motivadores del proceso, pues en las dos intervenciones al respecto más de la mitad lo mencionaron. Para la autoevaluación de la última semana se agregaron dos categorías más a estas tareas: ofrecer elementos que organizan la tarea y proponer estrategias, ambas con al menos 118 referencias.

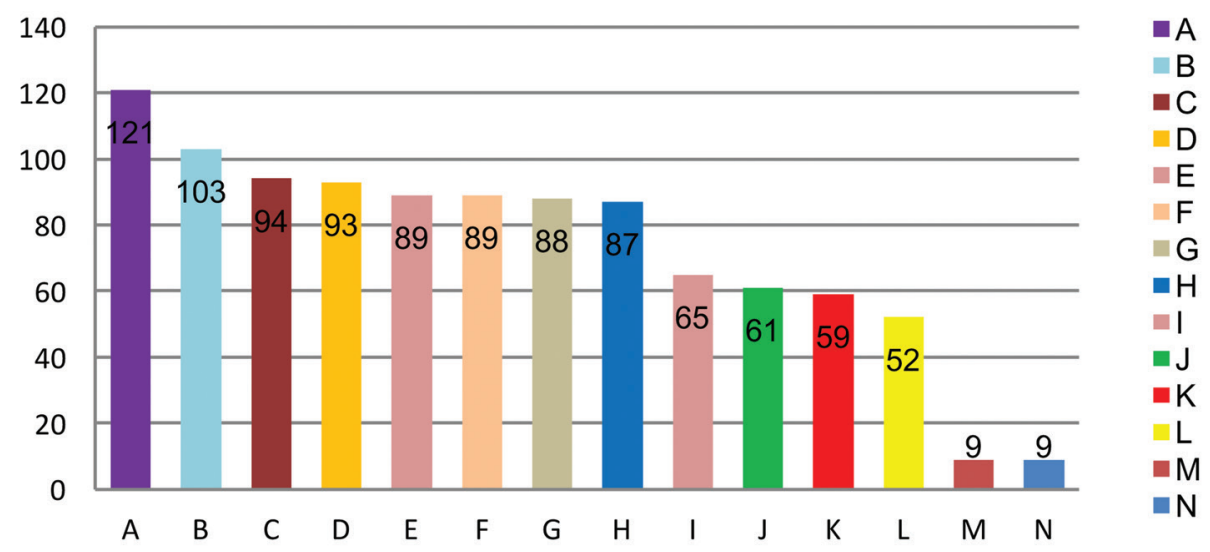

Figura 3. Intervenciones de los facilitadores para el aprendizaje (AA2).

Fuente: elaboración propia basada en los datos proporcionados por los participantes. 


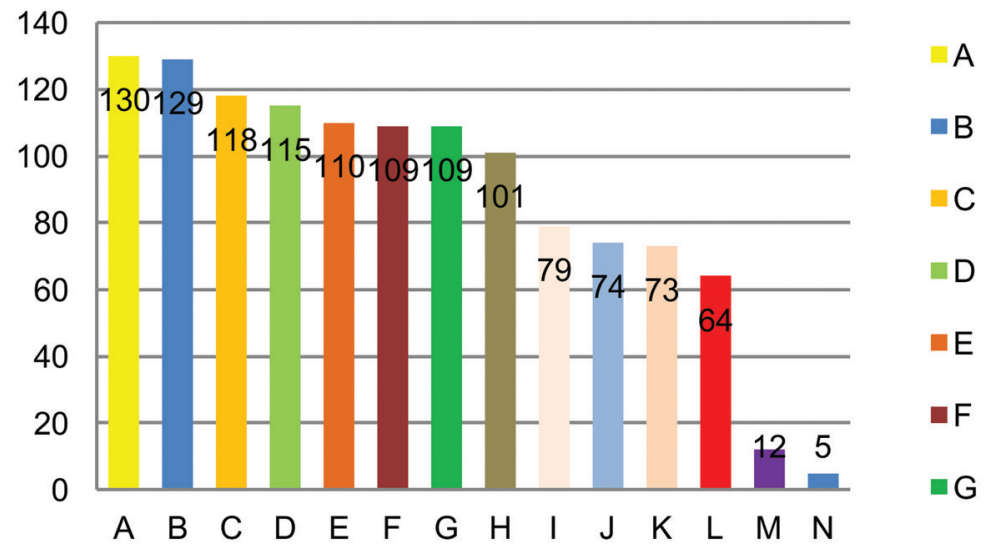

Figura 4. Intervenciones de los facilitadores para el aprendizaje (AA4).

Fuente: elaboración propia basada en los datos proporcionados por los participantes.

Con respecto a los facilitadores, se consultó acerca de las actividades que realizaron, esto se efectuó en la autoevaluación de la tercera semana (AA3); se pueden observar los resultados en la figura 5. Las categorías consideradas son las siguientes:

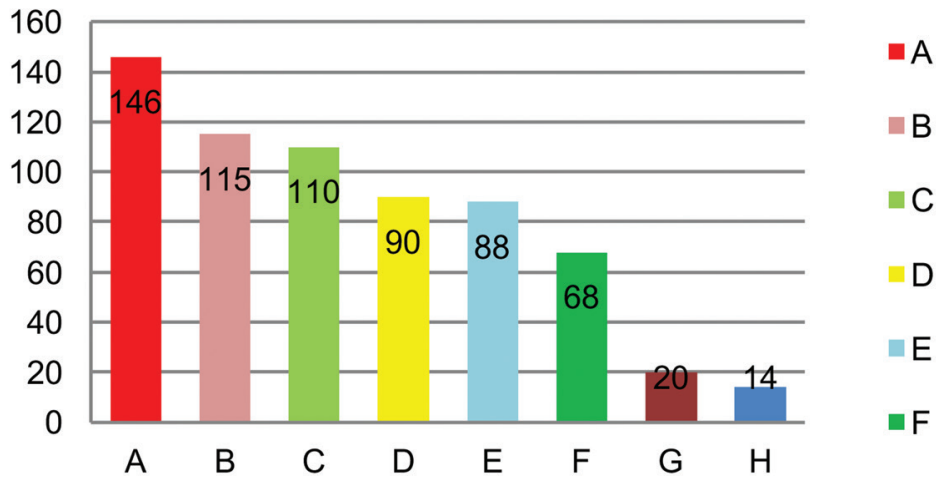

Figura 5. Actividades realizadas por los facilitadores (AA3).

Fuente: elaboración propia basada en los datos proporcionados por los participantes.

Las respuestas más repetidas fueron: el fomento del uso de tecnologías en los procesos de formación (bibliotecas digitales, bases de datos y tutoriales, entre otros) y las estrategias para que el estudiante interactúe con el entorno que investiga (contexto donde se realiza el estudio, con estudiantes, docentes, directivos y capacitadores, entre otros). La primera de las respuestas fue considerada por más de la mitad de los participantes y la segunda alcanza casi $50 \%$ de las respuestas. En ambos casos se observa el reconocimiento de que los facilitadores los motivaron para aprovechar no solo su entorno, sino las tecnologías a su disposición para apoyar la experiencia en el curso, lo cual aumenta las posibilidades de que transfieran sus aprendizajes a espacios y áreas más allá del curso. 


\section{Procesos de comunicación}

Los participantes identificaron la comunicación continua, la atención y el apoyo de los facilitadores como los procesos de comunicación que se mantuvieron entre ellos a lo largo del curso. Esta información se extrae tanto de la autoevaluación de la semana 4 (AA4) como de los participantes en los grupos presenciales, específicamente en el de Costa Rica.

Una muestra del valor que los participantes dieron a las estrategias de comunicación se aprecia en lo expresado en la actividad grupal de Costa Rica, acerca de los tutores académicos del curso:

"Algunos hasta se tomaron el tiempo para hacer capturas de pantalla y explicar cómo se hacía algo en la plataforma" [participación presencial, Costa Rica].

"Yo siempre buscaba las intervenciones de los TA porque solían ser las más atinadas" [participación presencial, Costa Rica].

Lo anterior evidencia que la comunicación de su parte fue acertada. Con respecto a la comunicación entre pares, fue evidente que se forjaron lazos que permitieron no solo hacer contactos académicos, sino aprender juntos. Ejemplos de esto son las siguientes intervenciones dadas en el mismo grupo presencial y en lo que se visualiza en la figura 6:

"Lo que nunca había hecho era compartir mis documentos, por ejemplo en Scribd, la conocía, nunca lo había hecho, es una red nueva para mí..." [participación presencial, Costa Rica].

"Entiendo lo de la evaluación por pares, era la única forma de evaluarnos a tanta gente".

Las categorías de la figura 6 son las siguientes:

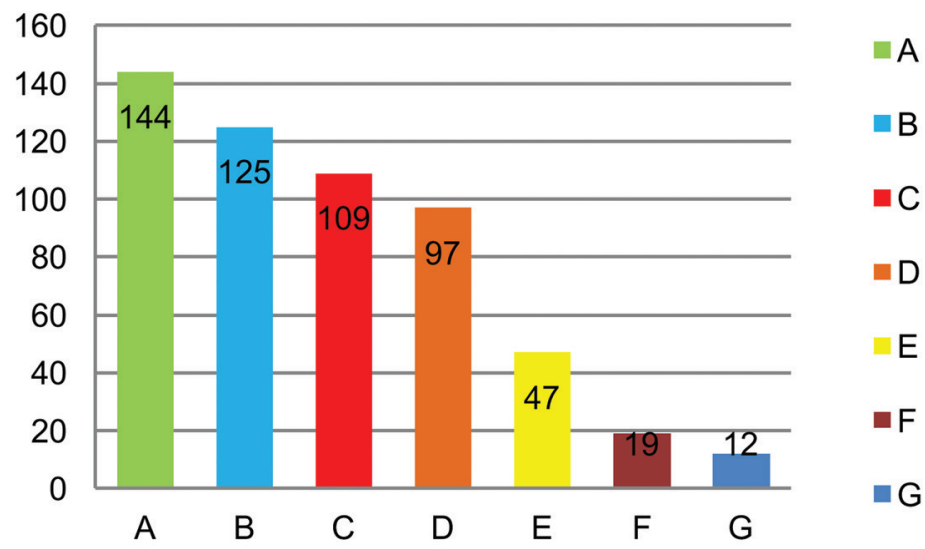

Figura 6. Procesos de comunicación detectados (AA4).

Fuente: elaboración propia basada en los datos proporcionados por los participantes.

Se muestran a continuación los resultados que se consideró contribuían a dar respuesta a la pregunta: ¿consideran los participantes que tienen posibilidades de transferir a su práctica diaria los aprendizajes obtenidos en el curso? Se muestran organizados según el elemento referido por la consulta, son los siguientes: expectativas, tipo de actividad mental, gestión del conocimiento, prácticas trabajadas y estrategias fomentadas y aplicaciones REA. 


\section{Expectativas}

Al empezar el curso, en la autoevaluación de la primera semana (AA1) se consultó a los participantes acerca de las expectativas que tenían.

Las respuestas obtenidas se organizaron en las siguientes categorías: usar REA, actualización, elaborar REA, uso de las TIC, compartir REA, mejorar práctica docente, aprender sobre los temas, establecer contactos profesionales, no responde. En la figura 7 es posible observar los resultados:

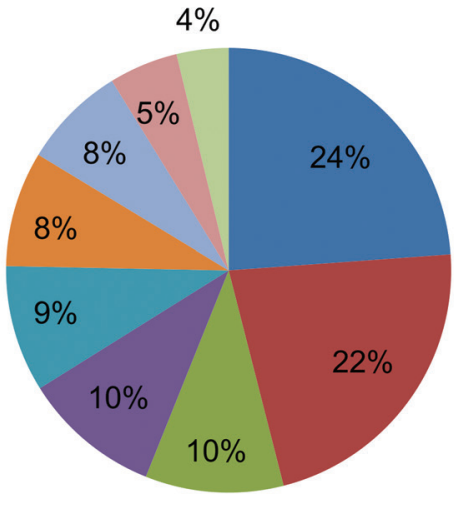

- Aprender sobre los temas

- Mejorar práctica docente

- Usar REA

- Actualización

No responde

- Uso de las TIC

Elaborar REA

Establecer contactos profesionales

Compartir REA

Figura 7. Expectativas iniciales (AA1).

Fuente: elaboración propia basada en los datos proporcionados por los participantes.

Observe que la categoría "Mejorar práctica docente", se convierte en la expectativa que obtiene la segunda mayor puntuación. De igual manera, las categorías "Usar REA", "Actualización", "Uso de las TIC", "Elaborar REA", y "Compartir REA" reflejan este deseo de transferir conocimientos a su propio quehacer, es decir, la transferencia forma parte de las expectativas de $62 \%$ de los participantes.

Al finalizar el curso de nuevo se cuestionaron las expectativas (en la autoevaluación de la semana 4, AA4), pero con un camino ya recorrido, ¿se cumplieron? El resultado fue el siguiente:

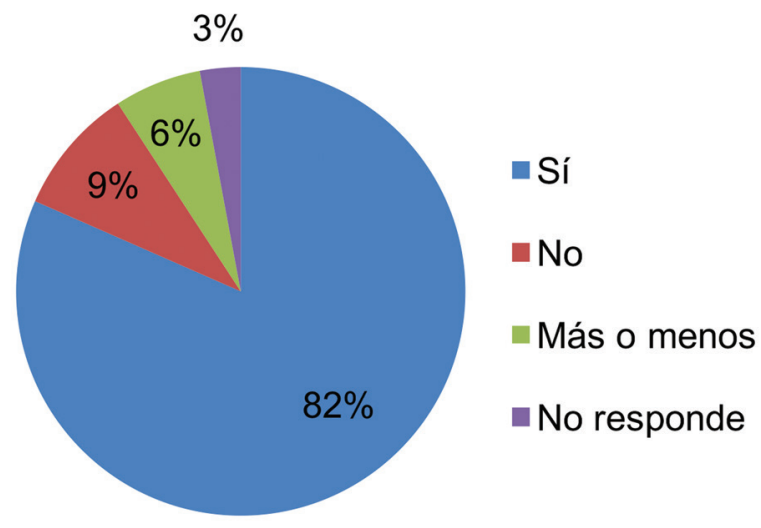

Figura 8. ¿Fueron satisfechas sus expectativas? (AA4).

Fuente: elaboración propia basada en los datos proporcionados por los participantes. 
Nótese que más de $88 \%$ consideraron que sus metas se cumplieron en alguna medida y menos de la décima parte aseguraron rotundamente que no fue así. Además, al relacionar las respuestas de los participantes al porqué de esta pregunta, con las expectativas que manifestaron en la pregunta anterior, es posible encontrar frases o explicaciones que apoyan la transferencia de los aprendizajes del curso a la práctica. lo anterior se encuentra, en gran medida, en las afirmaciones de los participantes tanto en la parte abierta de la autoevaluación de la semana 4 como en la sesión presencial de Costa Rica, algunas de sus impresiones son las siguientes:

"Sí, porque realmente aprendí más sobre los REA y me convencí de la utilidad de generar y compartir los recursos propios o que son productos de las clases que imparto."

"Sí, porque reforcé conocimientos que tenía y aprendí muchas otras cosas que me han ayudado en mi carrera profesional."

"Logré desarrollar herramientas a través del conocimiento adquirido" [participación presencial, Costa Rica].

"Obtuve información necesaria que puedo implementar en mi quehacer" [participación presencial, Costa Rica].

\section{Tipo de actividad mental}

En dos de las autoevaluaciones del curso, se consultó acerca de las actividades mentales trabajadas en el MOOC. Esto se llevó a cabo en la autoevaluación de la segunda semana (AA2) y posteriormente en la correspondiente a la cuarta semana (AA4). En la figura 9 se señala la actividad mental que más se trabajó según los participantes hasta la segunda semana. Las categorías de respuesta son las siguientes:

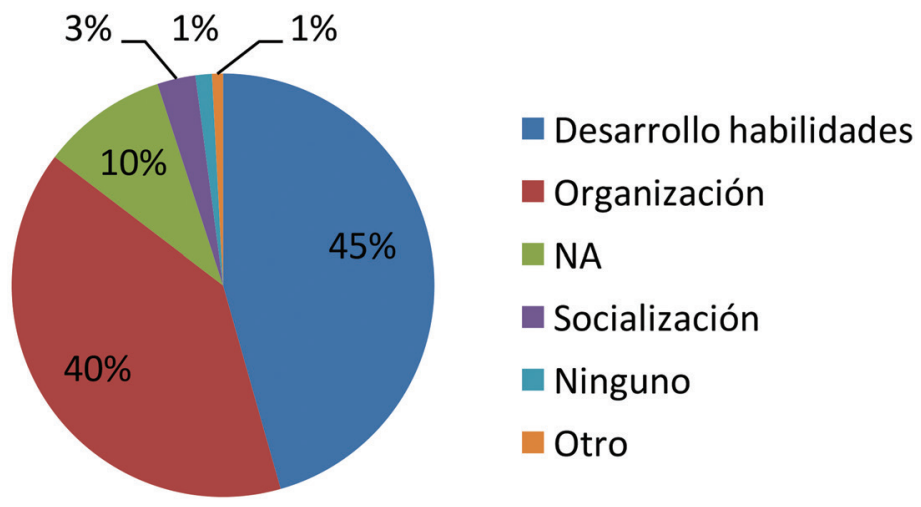

Figura 9. Tipo de actividad mental trabajada en el curso (AA2).

Fuente: elaboración propia basada en los datos proporcionados por los participantes.

Dos categorías son las predominantes: "Desarrollo de habilidades como el análisis, la síntesis, la conceptualización, la representación de información y conocimiento" con 45\% y "Organización y procesamiento de la información" con 40\%. Ambas son indispensables para la transferencia de conocimientos. Al finalizar el curso, en la autoevaluación de la cuarta semana (AA4), lo expresado se confirma con la percepción de los participantes de las actividades mentales que trabajaron arduamente en el curso. Obsérvese la figura 10, cuyas categorías son las siguientes: 


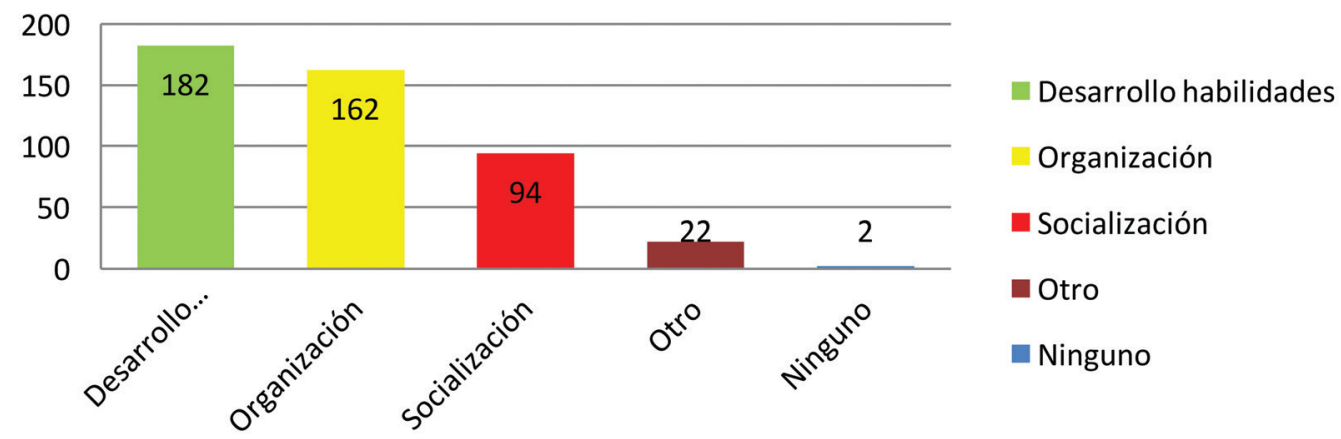

Figura 10. Tipo de actividad mental trabajada en el curso (AA4).

Fuente: elaboración propia basada en los datos proporcionados por los participantes.

Más de dos terceras partes de los participantes señalan que organizar y procesar información es una de las actividades mentales fomentadas en el curso y más de tres cuartas partes señalan que se desarrollaron habilidades mentales (análisis, síntesis, conceptualización, representación de información y conocimiento). También se encontraron datos de cómo los participantes en las sesiones presenciales hablaron del tema y dejaron claro que aprendieron conceptos, herramientas y estrategias que les serán de utilidad en su desarrollo profesional; por ejemplo:

"La hoja de ruta, o planeamiento para mí, me servirá para crear mis siguientes instrucciones de trabajo" [participación presencial, Costa Rica].

"De un tiempo para acá hay una explosión de materiales en línea, y este curso me abre los ojos a un nuevo tipo de materiales que pueden ser muy valiosos para la educación, que vamos a poder utilizar en clase o en la investigación que tenemos que realizar" [participante, presente en la sala, México].

"Nos deja muchas herramientas para evaluar" [participación presencial, Costa Rica].

\section{Gestión del conocimiento}

A los participantes se les consultó por el proceso de gestión del conocimiento que consideraron haber trabajado. La primera vez fue en la autoevaluación de la semana 3 (AA3), estos resultados se resumen en la figura 11 y las categorías son las siguientes:

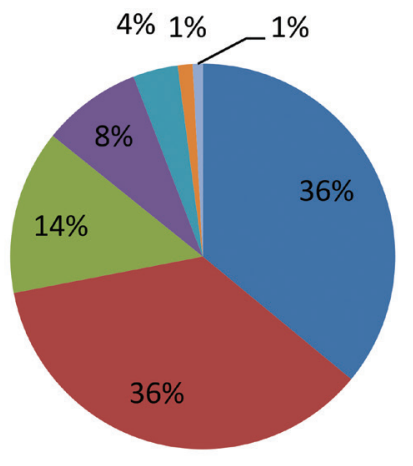

$$
\begin{aligned}
& \text { Acceso a información } \\
& \text { Reusabilidad del conocimiento } \\
& \text { Interacciones con el entorno } \\
& \text { NA } \\
& \text { Establecer conexiones } \\
& \text { Ninguno } \\
& \text { Otro }
\end{aligned}
$$

Figura 11. Proceso de gestión del conocimiento trabajado (AA3).

Fuente: elaboración propia basada en los datos proporcionados por los participantes. 
La gráfica denota que la mayor parte de los participantes eligieron el "acceso a la información y a los procesos de conocimiento" y "la reusabilidad del conocimiento que cotidianamente se comparte y se recrea"; juntos abarcan la opinión de $72 \%$ de los participantes. En vista de estas opiniones se puede asegurar que la propuesta potencia el proceso de transferencia de conocimiento analizado.

Al volver a consultar a los participantes acerca de estos procesos en la autoevaluación de la semana 4 (AA4), se consideraron todos los que valoraron para su caso particular, esta información se resume en la figura 12, las categorías son las siguientes:

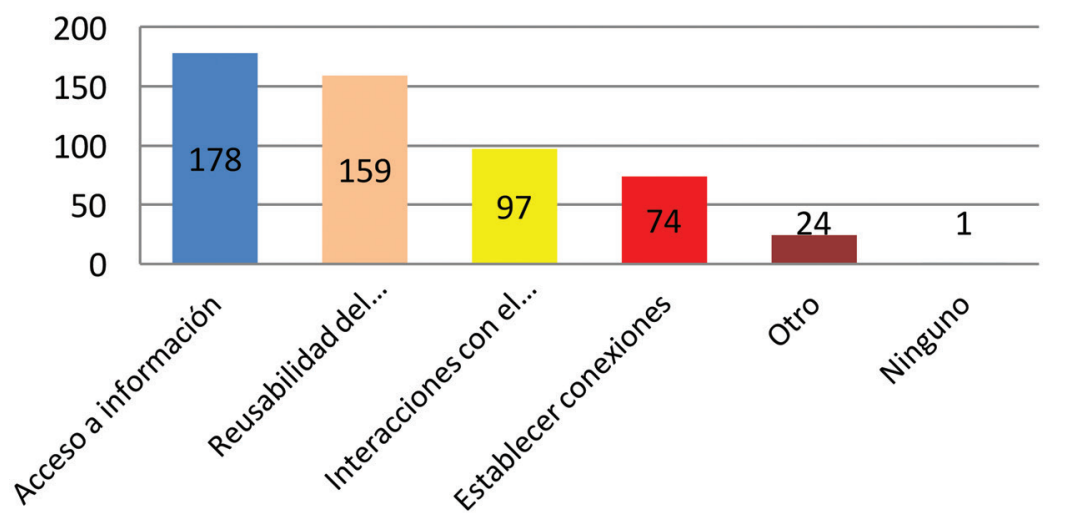

Acceso a información

Reusabilidad del

conocimiento

Interacciones con el entorno

Establecer conexiones

Otro

Ninguno

Figura 12. Proceso de gestión del conocimiento trabajado (AA4).

Fuente: elaboración propia basada en los datos proporcionados por los participantes.

Nuevamente las mismas categorías obtienen más de las dos terceras partes de las opiniones.

\section{Prácticas trabajadas y estrategias fomentadas}

Otra de las consultas hecha a los participantes fue acerca de las prácticas (tareas de aplicación semanal en el curso) que consideraban se trabajaron en el curso, tal pregunta se planteó en la autoevaluación de la semana 4 (AA4) y los resultados se resumen en la figura 13; las categorías son las siguientes:

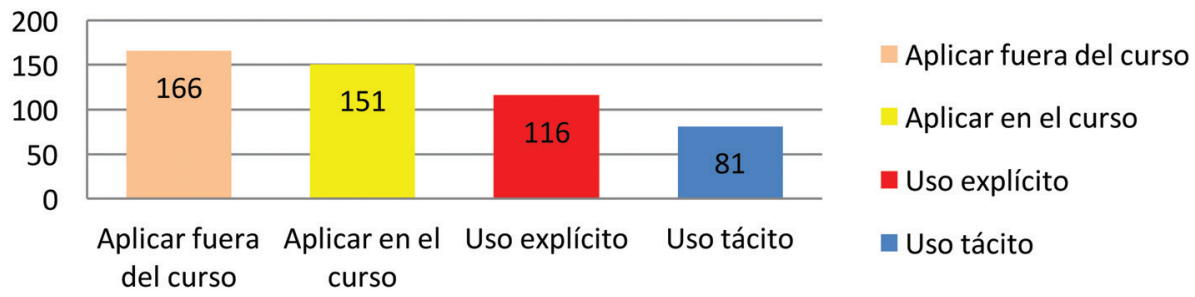

Figura 13. Prácticas trabajadas en el curso (AA4).

Fuente: elaboración propia basada en los datos proporcionados por los participantes. 
Los participantes eligieron, en su mayoría, que las prácticas realizadas en el MOOC fueron el fomento para aplicar fuera del curso lo aprendido (en contextos diversos), es decir, la transferencia. Esta respuesta proviene de casi $70 \%$ de los participantes. Como segunda opción eligieron el fomento para aplicar lo aprendido en el curso y fue mencionada por casi dos terceras partes de los consultados. Esto coincide con la expectativa que la mayoría definieron para el inicio del curso, en la cual indicaron que deseaban tener la posibilidad de aplicar lo del curso en su práctica.

Además al consultar por las estrategias fomentadas en el curso, coincidieron en las aplicaciones en la práctica educativa y las de difusión, lo cual se relaciona con lo afirmado respecto a la consulta de las prácticas trabajadas en el curso. En el área educativa, una participante en la sesión presencial de Costa Rica afirmó "puedo involucrar lo aprendido en mi desarrollo profesional".

\section{Aplicaciones de Recursos Educativos Abiertos (REA)}

A los participantes se les consultó directamente si ellos consideraban que podían emplear los REA en su práctica profesional, es decir, si podrían lograr la transferencia de conocimientos a su labor diaria. La consulta se llevó a cabo en dos momentos del curso, en la autoevaluación de la semana 1 y en la 4. La mayoría consideró que sí, y entre las opciones que eligieron como ejemplos de esas aplicaciones, la mayoría de los participantes en ambas consultas, consideraron las siguientes: para enriquecer mis clases (diseñar y/o preparar mi curso), para ofrecer material de referencia a mis alumnos (asignación de material de consulta), para realizar proyectos y propuestas innovadoras.

En la pregunta de la autoevaluación de la semana 4 había 10 respuestas posibles, 7 de las opciones tuvieron más de 119 menciones, es decir que más de la mitad de las personas de la muestra consideraron que era una manera para darle uso a los recursos.

Es interesante que las opciones elegidas por la mayoría no solo coincidieran en los dos momentos de consulta, sino que se evidencia que desean poner en práctica lo aprendido en el curso, en este caso, sobre los REA. Por otro lado, en las sesiones presenciales se destacaron las oportunidades y necesidades que se vislumbran a partir del curso. En Costa Rica se mencionó la necesidad de aprender más para construir REA y en México se indicó la potencialidad de estos recursos para enriquecer los cursos que se imparten en diferentes carreras.

\section{ANÁLISIS Y CONCLUSIONES}

La socialización es un elemento sustancial en los ambientes de aprendizaje de los cursos masivos abiertos. En los resultados del estudio destaca que la mayoría de los participantes coincidieron en que los foros y las redes sociales fueron sus lugares preferidos para compartir materiales y mejorarlos; asimismo, se observó que el MOOC cumplió al facilitar la socialización de conocimientos.

Según Kop et al. (2011) una de las grandes actividades de estos cursos es precisamente compartir el trabajo; lo que le asocia una importancia adicional a los espacios de socialización. Por tanto, se recomienda continuar abriendo espacios de comunicación sincrónica y asincrónica en donde los aprendices puedan compartir sus trabajos y recibir retroalimentación de sus pares para mejorarlos.

La variedad de actividades interactivas y formas de evaluación diferenciadas son elementos clave en los cursos masivos a distancia. En el estudio destacó que los aprendices consideraron valiosas las evaluaciones de pares y los portafolios como formas de evaluar los nuevos aprendizajes en un MOOC, además, comentaron que estas formas de evaluación les permitieron evidenciar la integración de sus conocimientos. 
Si se considera lo expuesto por Roig et al. (2014), la realimentación y las actividades de refuerzo bien valoradas por los participantes potencian mejores resultados respecto a la calidad pedagógica del curso; lo que permite visualizar que el diseño fue adecuado. Los portafolios y la evaluación de pares se pueden implementar en un MOOC como formas alternativas a la evaluación de un facilitador, esto permite a los participantes reflexionar acerca de lo que aprendieron en el curso.

En el diseño de los cursos masivos a distancia se debe planificar intencionadamente cómo los participantes pueden transferir los aprendizajes en ámbitos diferentes al curso. La transferencia de aprendizajes a través de la experiencia formativa del MOOC Innovación educativa con recursos abiertos se verifica manifiestamente en este estudio pues los participantes establecieron, como expectativa del curso, la transferencia de los aprendizajes a su práctica diaria, su desempeño laboral, profesional e incluso personal. En las consultas hechas a lo largo del curso sobre esto, se mantuvieron las respuestas afirmativas a esa posibilidad, tanto cuando se referían a los tipos de actividad mental, la gestión del conocimiento, las prácticas trabajadas, estrategias fomentadas y a las aplicaciones de los REA. Diseño y transferencia son conceptos que deben estar interrelacionados en las configuraciones de los ambientes de aprendizaje.

A continuación, se señalan las conclusiones puntuales para las dos preguntas que centraron la atención de este artículo.

a. ¿Cuáles son las percepciones de los participantes con respecto al diseño del curso?

Respecto a la socialización como un elemento del diseño del curso, la valoraron favorablemente. Kop et al. (2011) señalan que una de las grandes actividades de los MOOC es compartir el trabajo; lo que le imprime una importancia nueva a los espacios de socialización; por tanto se considera que se logra un buen trabajo en este aspecto.

La evaluación es otro elemento del diseño del ambiente trabajado en la investigación y se logró determinar que, según los participantes, sí se valoraron sus aprendizajes a lo largo del curso. Según Roig et al. (2014) esas metas claras, la realimentación y las actividades de refuerzo tan bien valoradas por ellos potencian mejores resultados respecto a la calidad pedagógica del curso; lo que evidentemente permite visualizar que el diseño fue adecuado.

Al considerar a los facilitadores, sus intervenciones y sus actividades como parte del diseño del curso, se visualizó que los participantes no solo valoraron su labor, sino que consideraron que aportaron más de lo esperado. Los autores Kop et al. (2011) afirman que los instructores son un elemento clave que permite el dinamismo en el curso y su intervención es fundamental para su éxito. En el caso del MOOC la labor de los TA fue un punto fuerte y clave para los logros que los participantes señalaron.

Por su parte, los procesos de comunicación (elemento transversal en el diseño) fueron altamente valorados por los participantes. Tanto Esposito (2012) como McAulay, Stewart y Siemens (2010) hacen hincapié en las condiciones para que la participación en un MOOC sea exitosa y se consiga un papel más activo de parte de las personas del curso. Es este caso, las condiciones estuvieron presentes y lograron el objetivo propuesto, por lo que el diseño de las mismas se considera apropiado.

Si se contemplan los resultados anteriores, es factible proponer el diseño de este curso MOOC como un modelo valioso y replicable para otros cursos de este tipo y hacer las adaptaciones necesarias según sea el área disciplinar del mismo.

b. ¿Consideran los participantes que tienen posibilidades de transferir a su práctica diaria los aprendizajes obtenidos en el curso?

Los participantes consideraban el curso como una oportunidad para mejorar su práctica. Al final del curso expresaron que pudieron cumplir este objetivo y que habían logrado aplicar los aprendizajes 
obtenidos. Así, los resultados alcanzados sugieren que los participantes del MOOC fueron capaces de transferir lo que aprendieron.

Este estudio apunta a que los participantes de MOOC muestran interés no solo en aprender sobre algún tema, sino en que el curso les brinde posibilidades y espacios para aplicar lo aprendido en su vida personal y profesional.

La investigación sobre estos elementos debe continuar ya que se necesitan MOOC más incluyentes y que promuevan cambios positivos en la vida personal, profesional y comunitaria de los participantes. Continuar con estudios de la transferencia en los $\mathrm{MOOC}$ es relevante para mejorar su diseño y facilitar la aplicación de los conocimientos generados. El presente estudio invita a seguir sumando aportes en el ámbito del movimiento educativo abierto y de la educación a distancia.

\section{REFERENCIAS}

Bhatti, M.; \& Kaur, S. (2010). The role of individual and training design factors on training transfer. Journal of European Industrial Training, 34(7), 656-672. Retrieved from www.scopus.com.

Cavanagh, S. (2013). MOOCs provider targets teacher education. Education Week, 32(31), 9. Retrieved from: http://connection.ebscohost.com/c/articles/87645436/moocs-provider-targets-teacher-education

Collins, K. (2003). Advanced sampling designs in mixed research: current practices an emerging trends in the social and behavioral sciences. In Tashakkori, A. \& Teddlie, C. (Eds.). Handbook of Mixed Methods in Social \& Behavioral Research (pp. 353-377). Thousand Oaks, CA: Sage.

Contreras, G.; Torres, R.; \& Ramírez, M. S. (2010). Uso de simuladores como recurso digital para la transferencia de conocimiento. Apertura Revista de Innovación Educativa, 2(1), 86.

Cormier, D.; \& Siemens, G. (2010). The Open Course: Through the Open Door--Open Courses as Research, Learning, and Engagement. Educause Review, 45(4), 30-32.

Creswell, J.; Klassen, A.; Plano, V. y Smith, K. (2011). Best practices for mixed methods research in the health sciences. Office of Behavioral and Social Sciences Research (OBSSR). Retrieved from http:// obssr.od.nih.gov/mixed_methods_research/pdf/Best_Practices_for_Mixed_Methods_Research. pdf

Creswell, J.; Plano, V.; Gutmann, M. y Hanson, W. (2003). Advanced mixed methods research design. En A. Tashakkori y C. Teddlie (Eds.). Handbook of mixed methods in social and behavioral research (pp. 209-240). Thousand Oaks, CA: Sage.

Creswell, J. y Plano, V. (2011). Designing and conducting Mixed Method Research. Thousand Oaks, CA: Sage.

De la Cuesta, C. (2006). La teoría fundamentada como herramienta de análisis. Cultura de los cuidados, 20(2), 136-140.

Esposito, A. (2012). Research ethics in emerging forms of online learning: Issues arising from a hypothetical study on a MOOC. Electronic Journal of e-Learning, 10(3), 315-325. Recuperado de: www.scopus. com.

Gegenfurtner, A. (2011). Motivation and transfer in professional training: a meta-analysis of the moderating effects of knowledge type, instruction, and assessment conditions. Educational Research Review, 6(3), 153-168. Retrieved from www.scopus.com.

Gómez, M. (2011). Elementos de estadística descriptiva. San José: Editorial de la Universidad Estatal a Distancia. 
Kop, R., Fournier, H. y Mak, J. (2011). A pedagogy of abundance or a pedagogy to support human beings? participant support on massive open online courses. International Review of Research in Open and Distance Learning, 12(7) Special Issue, 74-93. Recuperado de www.scopus.com.

López, A. (s.f.). Cálculo del tamaño de una muestra. Universidad Autónoma de Madrid. Recuperado de: www.uam.es/personal_pdi/economicas/amlopez/n_optimo.xls.

McAulay, A., Stewart, B. y Siemens, G. (2010). The MOOC model for digital practice. University of Prince Edward Island. Recuperado de: http://www.elearnspace.org/Articles/MOOC_Final.pdf.

Ramírez, María S. (2012). Modelos y estrategias de enseñanza para ambientes innovadores. México: Editorial digital. Tecnológico de Monterrey.

Roig, R., Mengual, S. y Suárez, C. (2014). Evaluación de la calidad pedagógica de los MOOC. Profesorado. Revista del currículum y formación del profesorado, 18(1), 28-41. Recuperado de: http://www.ugr. es/ recfpro/rev181ART2res.pdf.

Sandoval, C. (2002). Investigación cualitativa. Programa de especialización en teoría, métodos y técnicas de investigación social. Bogotá: ICFES.

Siemens, G. (2008). Learning and knowing in networks: Changing roles for educators and designers. ITFORUM for Discussion. Recuperado de: http://www.ingedewaard.net/papers/connectivism/2008_ siemens_Learning_Knowing_in_Networks_changingRolesForEducatorsAndDesigners.pdf.

Strauss, A. y Corbin, J. (1997). Grounded theory in practice. Thousand Oaks, CA: Sage.

Wenzelburger, E. (1987). La transferencia en el aprendizaje. Revista de la Educación Superior, XVI(1)(61), 1-10.

Volet, S. (2013). Extending, broadening and rethinking existing research on transfer of training. Educational Research Review, 8, 90-95. Retrieved from www.scopus.com. 
